\title{
Low energy phenomenology of the overdoped cuprates: Viability of the Landau-BCS paradigm
}

\author{
N. R. Lee-Hone $\odot,{ }^{1}$ H. U. Özdemir, ${ }^{1}$ V. Mishra,,${ }^{2, *}$ D. M. Broun, ${ }^{1,3}$ and P. J. Hirschfeld ${ }^{4}$ \\ ${ }^{1}$ Department of Physics, Simon Fraser University, Burnaby, British Columbia, Canada V5A 1S6 \\ ${ }^{2}$ Computer Science and Mathematics Division, Oak Ridge National Laboratory, Oak Ridge, Tennessee 37831, USA \\ ${ }^{3}$ Canadian Institute for Advanced Research, Toronto, Ontario, Canada MG5 $1 Z 8$ \\ ${ }^{4}$ Department of Physics, University of Florida, Gainesville, Florida 32611, USA
}

(Received 21 February 2019; revised manuscript received 4 September 2019; accepted 25 November 2019; published 28 February 2020; corrected 3 August 2021)

\begin{abstract}
We use dirty $d$-wave BCS theory to calculate absolute superfluid density, residual specific heat, Volovik effect, and thermal conductivity and compare to experiments on the cuprate superconductor $\mathrm{La}_{2-x} \mathrm{Sr}_{x} \mathrm{CuO}_{4}$, showing that the theory provides a surprisingly good account of the data across the overdoped region. The starting point is an empirical angle-resolved photoemission spectroscopy-based parametrization of the electronic structure, including substantial Fermi-liquid renormalizations. Furthermore, a proper treatment of the less-explored weak out-of-plane dopant disorder limit is found to be essential. We then show that the same approach captures the low energy physics of another important overdoped cuprate, Tl-2201, thought to be much "cleaner" since it exhibits quantum oscillations, low residual resistivities, and small superconducting state Sommerfeld coefficients. We conclude that the low energy properties of cuprates are remarkably well described in the overdoped regime by dirty $d$-wave theory, without the need to introduce physics beyond the Landau-BCS paradigm.
\end{abstract}

DOI: 10.1103/PhysRevResearch.2.013228

\section{INTRODUCTION}

Of the various phases observed in the hole-doped cuprate phase diagram [1], the one which seems most conventional in many ways is the $d$-wave superconductor between hole concentrations of roughly $p_{c 1} \simeq 5 \%$ and $p_{c 2} \simeq 30 \%$. Over much of this range, from onset to slightly past optimal doping, superconductivity condenses out of a state that is poorly understood, characterized by a pseudogap in the one-particle spectrum [2], unusual transport properties [3-5], and several other coexisting symmetry-breaking orders [6-9]. On the other hand, if one is able to dope into the so-called overdoped region, most of these effects seem to disappear and one can imagine solving the much simpler problem of $d$-wave superconductivity condensing out of a Fermi liquid.

Shortly after the discovery of cuprate superconductivity, the existence of $d$-wave superconductivity did not seem likely at all, as it was widely believed that disorder would destroy higher angular momentum pair states [10]. Doping takes place for the most part via chemical substitution or oxygen removal in various layers away from the $\mathrm{CuO}_{2}$ planes and in the process almost always introduces disorder. In the early 1990 s, studies showed theoretically that the presence of small amounts of disorder in cuprates was both compatible with the existence of the $d$-wave state (particularly because dopants created rather weak scattering potentials) and also rather

\footnotetext{
*Present address: Kavli Institute for Theoretical Sciences, University of Chinese Academy of Sciences, Beijing 100190, China.

Published by the American Physical Society under the terms of the Creative Commons Attribution 4.0 International license. Further distribution of this work must maintain attribution to the author(s) and the published article's title, journal citation, and DOI.
}

important for understanding the observed properties [11-14]. This theoretical approach, similar to the Abrikosov-Gor'kov theory of disordered conventional superconductors [15], now goes under the name of dirty $d$-wave theory. Still, most discussions of the overdoped phases have ignored these effects, focusing instead on possible intrinsic physics despite the fact that overdoping by chemical substitution necessarily introduces higher concentrations of impurities.

A key question in this context is the mechanism responsible for the reduction in $T_{c}$ on overdoping and the disappearance of superconductivity at $p_{c 2}$. For the most part, this has been attributed to intrinsic effects: It has been assumed that the strength of the pairing correlations in the $\mathrm{CuO}_{2}$ plane weakens as one goes to higher doping [16], due to both the enhanced screening of the local Coulomb interaction and the easing of the Mott constraint on hole kinematics. However, a few authors have discussed the role of disorder in suppressing $T_{c}$ [17], and this scenario was particularly emphasized by Rullier-Albenque et al. [18], who pressed the analogy between systematic irradiation disorder, which suppresses $p_{c 2}$ in $\mathrm{YBa}_{2} \mathrm{Cu}_{3} \mathrm{O}_{6+y}$, and dopant disorder.

Another longstanding puzzle is the apparent contradiction between the "universal" physics of the $\mathrm{CuO}_{2}$ planes [1,19] and the wide variation of maximum $T_{c}$ 's across cuprate families. An obvious and strong correlation is the number of planes per unit cell, but even within the single-layer cuprates the maximum $T_{c}$ varies from roughly $10 \mathrm{~K}$ for Bi-2201 to $40 \mathrm{~K}$ for $\mathrm{La}_{2-x} \mathrm{Sr}_{x} \mathrm{CuO}_{4}$ (LSCO) to $90 \mathrm{~K}$ for $\mathrm{Hg}-1201$ and $\mathrm{Tl}-2201$. Some authors have provided explanations in terms of band structure differences driven by apical oxygen states [20,21] or admixtures of additional $\mathrm{Cu} d$ orbitals at the Fermi surface of different materials [22]. However it is also true that various single-layer cuprates are doped in rather dissimilar ways. Fujita et al. [23] proposed that the strength of the potential 
scattering introduced by the dopants in individual cuprates might account for most of the $T_{c}$ variation.

In this paper we show that low energy properties of overdoped cuprates in two disparate but archetypical materials can be understood using only standard $d$-wave BCS and Landau Fermi-liquid theory. The remarkable agreement found between theory and experiment depends on disorder effects being properly treated and on a realistic parametrization of the electronic structure, including substantial Fermi-liquid renormalizations. Our conclusions contradict those of recent experimental studies of superfluid density and optical conductivity on high quality LSCO films [24,25]. Starting from the same dirty $d$-wave framework and disorder parameters used previously to study the relative temperature dependence of superfluid density [26] and terahertz (THz) conductivity [27] in LSCO, we show that the absolute superfluid density, residual specific heat, Volovik effect, and thermal conductivity can also be understood in LSCO. We then extend the comparison to the Tl-2201 system, another single-layer cuprate that can be tuned throughout the overdoped regime. The latter step provides a particularly stringent test of the model, as Tl-2201 is thought to be a very clean system, due to its manifestation of quantum oscillations [28-30] and the observation of clean-limit behavior in other properties [31-33]. We show that despite being indeed significantly cleaner than LSCO, overdoped Tl-2201 is describable within the same dirty $d$-wave framework and is sufficiently dirty to display the same unusual non-BCSlike proportionality of superfluid density to $T_{c}$, as recently measured in overdoped LSCO [24].

\section{DIRTY $d$-WAVE THEORY}

The history and structure of the dirty $d$-wave theory have been reviewed recently in Ref. [27]. It is based on the singleparticle Green's function

$$
G\left(\mathbf{k}, i \omega_{n}\right)=-\frac{i \tilde{\omega}_{n} \tau_{0}+\Delta_{\mathbf{k}} \tau_{1}+\xi_{\mathbf{k}} \tau_{3}}{\tilde{\omega}_{n}^{2}+\Delta_{\mathbf{k}}^{2}+\xi_{\mathbf{k}}^{2}}
$$

where $\Delta_{\mathbf{k}}$ is the $d$-wave superconducting gap at wave vector $\mathbf{k}, \xi_{\mathbf{k}}$ is the single-particle energy, $\tau_{i}$ are the Pauli matrices in Nambu space, and $\tilde{\omega}_{n}$ is a renormalized Matsubara frequency that, in the self-consistent $t$-matrix approximation [34,35], obeys

$$
\tilde{\omega}_{n}=\omega_{n}+i \Sigma_{\mathrm{imp}}\left(\tilde{\omega}_{n}\right)=\omega_{n}+\pi \Gamma \frac{\left\langle N_{\mathbf{k}}\left(\tilde{\omega}_{n}\right)\right\rangle_{\mathrm{FS}}}{c^{2}+\left\langle N_{\mathbf{k}}\left(\tilde{\omega}_{n}\right)\right\rangle_{\mathrm{FS}}^{2}} .
$$

Impurity scattering is assumed to be characterized by parameters $(\Gamma, c)$, where $c$ is the cotangent of the scattering phase shift and $\Gamma$ is a scattering rate parameter proportional to the concentration of impurities. The corresponding normal-state scattering rate is $\Gamma_{N}=\pi \Gamma /\left(1+c^{2}\right)$. In addition, $N_{\mathbf{k}}\left(\tilde{\omega}_{n}\right)=$ $\tilde{\omega}_{n} /\left(\tilde{\omega}_{n}^{2}+\Delta_{\mathbf{k}}^{2}\right)^{1 / 2}$ is the integrated diagonal Green's function and $\langle\cdots\rangle_{\mathrm{FS}}$ is a Fermi-surface average, defined by Eqs. (A1) and (A2). The critical temperature and the temperature dependence of the order parameter are obtained by solving the gap equation

$$
\Delta_{\mathbf{k}}=2 \pi T N_{0} \sum_{\omega_{n}>0}^{\omega_{0}}\left\langle V_{\mathbf{k}, \mathbf{k}^{\prime}} \frac{\Delta_{\mathbf{k}^{\prime}}}{\sqrt{\tilde{\omega}_{n}^{2}+\Delta_{\mathbf{k}^{\prime}}^{2}}}\right\rangle_{\mathrm{FS}},
$$

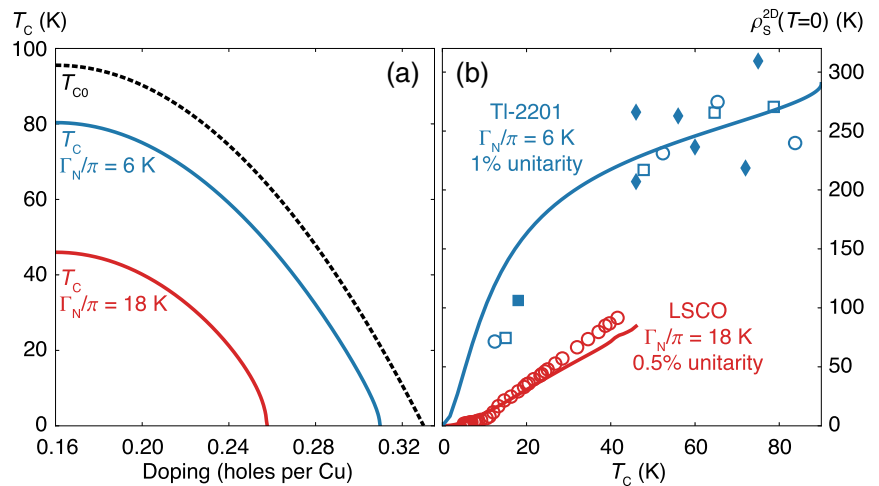

FIG. 1. Predictions of dirty $d$-wave theory. (a) For a single parabolic doping dependence of the underlying $T_{c 0}(p)$, different choices of $\Gamma_{N}$ result in superconducting domes $T_{c}(p)$ reminiscent of Tl-2201 and LSCO. (b) Starting from accurate parametrizations of the Fermi surfaces, the same theory captures the strong correlation between $\rho_{s}$ and $T_{c}$ observed in experiment. LSCO data: O, molecular-

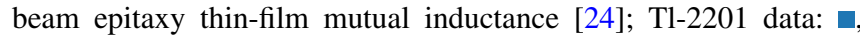
single-crystal microwave [37]; $\diamond$, single-crystal muon spin relaxation ( $\mu$ SR) [38]; $\mathrm{O}$ and $\square$, polycrystalline $\mu$ SR $[39,40]$.

where $N_{0}$ is the total density of states, $V_{\mathbf{k}, \mathbf{k}^{\prime}}$ is the $d$-wave pairing interaction, and $\omega_{0}$ is a high energy cutoff.

\section{COMPARISON OF LSCO AND TI-2201}

In Refs. [24,25], arguments were given why disorder could not be the cause of the unusual superconducting behavior observed in overdoped LSCO films. Principal among these were the linearity of the penetration depth measured to relatively low temperatures, but this turns out to be a feature of $d$-wave superconductors in the presence of weak scatterers, as pointed out in Refs. [26,36] and plotted in Fig. 5. As discussed in Appendix C, the Born limit is adequate to discuss impurity potentials $V_{\text {imp }} \lesssim 0.1 \mathrm{eV}$, i.e., $c \gtrsim 2$, which we believe includes out-of-plane chemical substituents and interstitials in cuprates. Furthermore, a disorder-based explanation of the properties of overdoped LSCO is perhaps not unexpected, since many other characteristics of this system are consistent with dirty limit behavior, e.g., the failure to observe quantum oscillations.

As mentioned above, by many measures Tl-2201 is a much cleaner cuprate system. However, it has been established for some time that the superfluid density correlates strongly with $T_{c}$, as expected only in a dirty BCS superconductor [37-40]. It therefore behooves us to consider the Tl-2201 system as an important test of dirty $d$-wave theory and its ability to explain the nonuniversal aspects of the overdoped cuprate families.

First, we ask, how clean is Tl-2201 really? Estimates of the Dingle temperature in a $T_{c}=27 \mathrm{~K}(p=0.27)$ sample give a single-particle electronic mean free path in the normal state of about $360 \AA$ [30], compared to a transport mean free path of $620 \AA$ from microwave measurements [37]. This suggests a modest amount of forward-scattering character of the out-of plane defects in the Tl system, which we ignore for the moment but will discuss below. By contrast, the transport mean free path deduced from the Mahmood et al. [25] terahertz measurements on LSCO is of order $100 \AA$, 5-6 times 

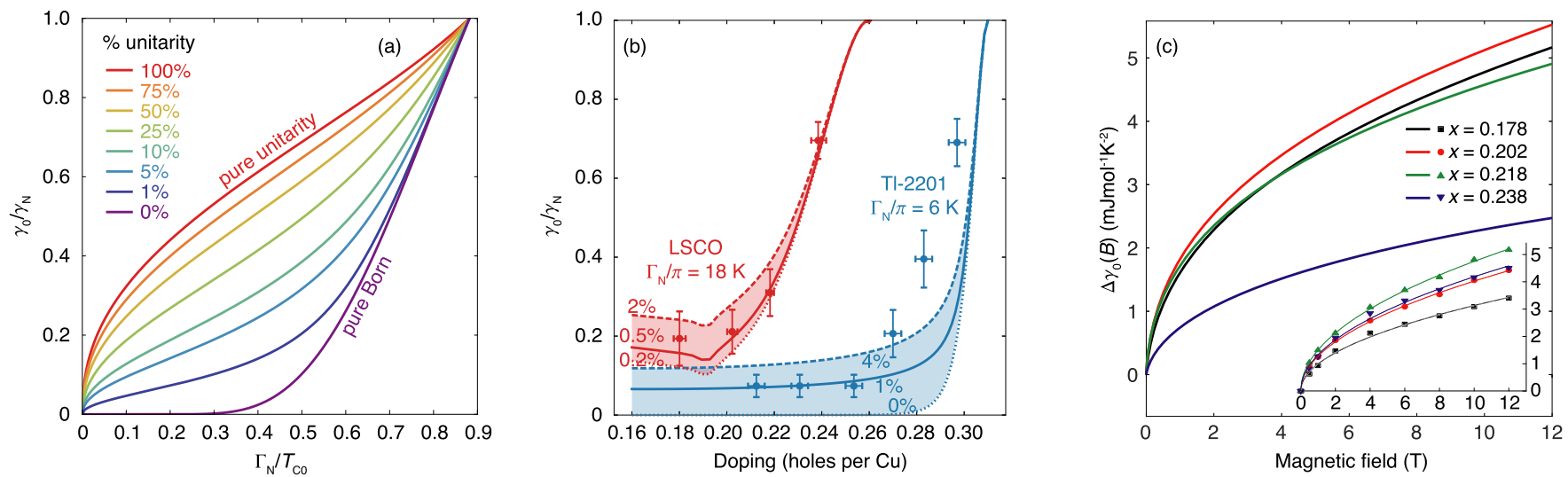

FIG. 2. Specific heat in the dirty $d$-wave theory. (a) The residual Sommerfeld coefficient $\gamma_{0}$ is a particularly sensitive probe of the degree of unitarity scattering in a $d$-wave superconductor. Curves are parametrized by the percentage contribution unitarity scattering makes to the normal-state scattering rate $\Gamma_{N}$. (b) Comparison of $d$-wave theory with zero-field heat capacity data for LSCO [44] and Tl-2201 [45]. (For Tl-2201, $\gamma(T)$ has been extrapolated to $T=0$ using an equal entropy construction; see Appendix D.) Errors bars denote experimental uncertainties. For fixed total normal-state scattering rate $\Gamma_{N}$, dirty $d$-wave results are plotted for varying percentages of unitarity scattering, as indicated. (c) Calculated field dependence of residual Sommerfeld coefficient in the Doppler-shift approximation, for a square vortex lattice in LSCO [46], at four different dopings, for $\Gamma_{N} / \pi=18 \mathrm{~K}$ and $0.5 \%$ unitarity scattering, exhibiting the Volovik effect, $\Delta \gamma \sim \sqrt{H}$. The inset shows the corresponding experimental data from Ref. [44].

smaller than in Tl-2201. This factor is roughly consistent with comparisons of scattering rates in the two systems by Bangura et al. [29] and also qualitatively consistent with the proposal of Fujita et al. [23] that those materials with A-site dopant disorder suffer from more pair breaking than those where dopants reside in an additional layer distant from the $\mathrm{CuO}_{2}$ plane. Experimentally, it is known that the dominant source of cation disorder in Tl-2201 is an approximately $10 \%$ excess of $\mathrm{Cu}$ that substitutes for $\mathrm{Tl}$ [41]. The $\mathrm{TlO}$ layers in which this disorder resides are approximately 3 times further from the $\mathrm{CuO}_{2}$ planes than the $\mathrm{LaO}$ layers in LSCO in which the dopant $\mathrm{Sr}$ atoms reside.

We therefore examine the predictions of the dirty $d$-wave theory for weak to intermediate dopant-type disorder, in a system several times cleaner than LSCO. Although, as discussed in the Introduction, there are many factors that appear to contribute to the maximum $T_{c}$ of various cuprates, it is instructive to examine the effect of disorder in isolation. In Fig. 1(a) we show how two very different $T_{c}(p)$ relations, corresponding approximately to those of Tl-2201 and LSCO, can emerge from a single clean-limit reference system (single $T_{c 0}(p)$ curve), using the Abrikosov-Gor'kov $T_{c}$ suppression, with $\Gamma_{N} / \pi=18 \mathrm{~K}$ for the LSCO-like curve, as in earlier work $[26,27]$ and $\Gamma_{N} / \pi=6 \mathrm{~K}$ for Tl-2201. Note that a larger critical doping naturally emerges for Tl-2201 $\left(p_{c 2}=\right.$ $0.31)$ than for LSCO $\left(p_{c 2}=0.26\right)$, in accord with experiment [28-30].

In Fig. 1(b) we plot the two-dimensional superfluid density

$$
\rho_{s}^{2 \mathrm{D}}(T)=\frac{\hbar^{2} d}{4} 2 \pi T N_{0} \sum_{\omega_{n}>0}\left\langle v_{F, x}^{2} \frac{\Delta_{\mathbf{k}}^{2}}{\left(\tilde{\omega}_{n}^{2}+\Delta_{\mathbf{k}}^{2}\right)^{3 / 2}}\right\rangle_{\mathrm{FS}},
$$

calculated using the LSCO disorder parameters from Ref. [26], together with the same calculation for parameters appropriate for $\mathrm{Tl}-2201$. The tight-binding parametrizations of the Fermi surfaces $[42,43]$ are discussed in Appendix B. We note in particular that the Tl-2201 parametrization is based directly on low energy angle-resolved photoemission spectroscopy (ARPES) and therefore requires no additional renormalization of the dispersion. It is seen that the dirty $d$-wave model describes both the cleaner Tl-2201 system and the dirty LSCO system quite well, nevertheless capturing the observed non-BCS scaling of $\rho_{s}$ with $T_{c}$ in the Tl-system despite this behavior being associated with significant disorder in BCS theory.

Since cuprates are famous for displaying behavior that deviates strongly from that of Fermi liquids, making a case for a conventional description in the overdoped region of the phase diagram requires further testing and comparison with additional data. In Fig. 2 we display the results of an evaluation of the superconducting state specific heat $C(T)=T d S / d T$, obtained from the Bogoliubov quasiparticle entropy [47]

$$
S=-k_{B} \int d \omega N(\omega)[f \ln f+(1-f) \ln (1-f)],
$$

where $f=f(\omega, T)$ is the Fermi function. In Fig. 2(a) we illustrate how the residual Sommerfeld coefficient $\gamma_{0}=$ $\lim _{T \rightarrow 0} C(T) / T$, reflecting the density of states $N(0)$, depends on scattering phase shift and is dominated mostly by the strong scatterers. This comparison is thus the most sensitive way to pin down the magnitude of the near-resonant disorder scattering in the $\mathrm{CuO}_{2}$ planes. In Fig. 2(b) we present comparisons of the theory with existing data on doping-dependent Sommerfeld coefficients of LSCO [44] and Tl-2201, where the latter have been obtained using entropy-conserving fits to the data in Ref. [45], as described in Appendix D. The appropriate values of the unitarity limit scattering rate parameter are somewhat smaller than used in our earlier comparisons: this does not change any of the fits or conclusions of those studies, as the electromagnetic response is far less sensitive to strong scattering than the heat capacity. Overall, the dirty $d$-wave model fits the doping dependence of the experimental data on both systems extremely well. 


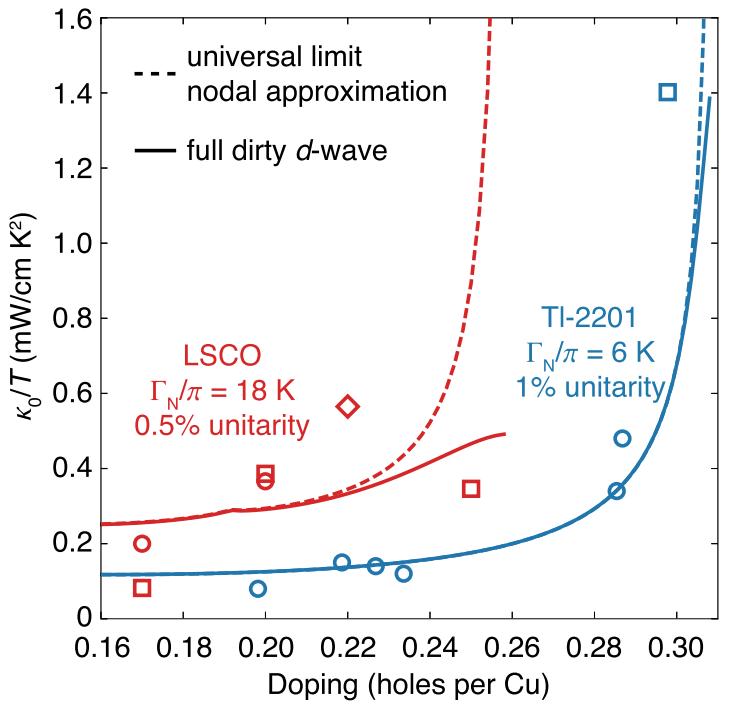

FIG. 3. Thermal conductivity data on LSCO (O [51] and $\square$ [52]) and Tl-2201 (O [53] and $\mathrm{[32])} \mathrm{compared} \mathrm{to} \mathrm{dirty} d$-wave theory with appropriate parameters, as discussed in the text. Note the breakdown of the universal-limit nodal approximation on the approach to $p_{c 2}$ in LSCO, but not in Tl-2201.

The effect of a magnetic field in the superconducting state can be approximated by including the Doppler shift of the quasiparticle energy in the semiclassical approximation to the density of states [48-50],

$$
N(\omega, H)=N_{0} \operatorname{Im}\left\langle\left\langle\frac{\tilde{\omega}\left(\omega-\frac{1}{2} \mathbf{p}_{s} \cdot \mathbf{v}_{\mathbf{k}}\right)}{\sqrt{\tilde{\omega}^{2}\left(\omega-\frac{1}{2} \mathbf{p}_{s} \cdot \mathbf{v}_{\mathbf{k}}\right)-\Delta_{\mathbf{k}}^{2}}}\right\rangle\right\rangle_{\mathrm{FS}} .
$$

Here $\mathbf{p}_{s}$ is the local gauge-invariant superfluid momentum at point $R$ and \langle\rangle$_{R}$ is a spatial average over the vortex lattice unit cell, as described in detail in Appendix E. In Fig. 2(c) we show the calculated field dependence of the superconducting state Sommerfeld coefficient for a range of LSCO dopings, with the same impurity parameters used above. The most striking aspect is the persistence of the approximate $\gamma \sim \sqrt{H}$ behavior (the so-called Volovik effect) in relatively dirty samples. In addition, the overall magnitude of the field variation is quite consistent with the experimental data from Ref. [44]. In the original paper, by focusing on the strong scattering regime of the dirty $d$-wave theory, the experimentalists were unable to reconcile the relatively large $\gamma_{0}$ with the observation of apparently clean-limit $\sqrt{H}$ behavior and concluded that the system must be undergoing real-space phase separation. In fact, we see here that this apparent inconsistency is explained naturally when one accounts for the presence of mostly weak scatterers and a small concentration at the unitarity limit.

Next we discuss the thermal conductivity in the superconducting state, a sensitive probe of the lowest energy mobile $d$-wave quasiparticles, with

$$
\begin{aligned}
\frac{\kappa}{T}= & \frac{N_{0}}{2} \int_{0}^{\infty} d \omega \frac{\omega^{2}}{T^{2}} \frac{\partial f}{\partial \omega} \\
& \times\left\langle\frac{v_{F, x}^{2}}{\operatorname{Re} \sqrt{\tilde{\Delta}_{\mathbf{k}}^{2}-\tilde{\omega}^{2}}}\left[\frac{\left|\tilde{\Delta}_{\mathbf{k}}\right|^{2}-|\tilde{\omega}|^{2}}{\left|\tilde{\Delta}_{\mathbf{k}}^{2}-\tilde{\omega}^{2}\right|}-1\right]\right\rangle_{\mathrm{FS}},
\end{aligned}
$$

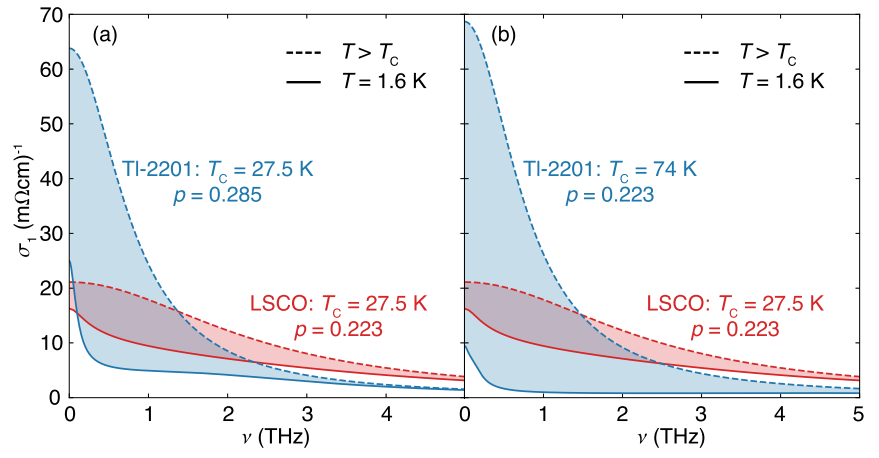

FIG. 4. Comparison of the optical conductivity of overdoped LSCO and Tl-2201, calculated in the dirty $d$-wave model for (a) materials with the same $T_{c}$ but different doping level and (b) materials with the same doping level but different $T_{c}$. Shaded regions denote the spectral weight that condenses to form the low temperature superfluid. Impurity parameters are the same as earlier in the paper: $\Gamma_{N} / \pi=6 \mathrm{~K}$ for Tl-2201, with $1 \%$ unitarity scattering, and $\Gamma_{N} / \pi=$ $18 \mathrm{~K}$ for LSCO, with $0.5 \%$ unitarity scattering.

which reduces at low $T$ and $\Gamma_{N}$ to the universal limit $\kappa_{00} / T \equiv$ $k_{B}^{2} v_{F} / 3 \hbar d v_{\Delta}$, where the gap velocity $v_{\Delta}$ includes the effects of disorder via self-consistent solution of the BCS gap equation (3). In Fig. 3 we compare evaluations of Eq. (7) for the same LSCO and Tl-2201 disorder parameters and band structures to the available experimental data. Note that the full theory distinguishes between the rapid rise of $\kappa_{0} / T$ as $p \rightarrow$ $p_{c 2}$ in Tl-2201 and the weak doping dependence in LSCO. The consistency is particularly impressive given the lack of adjustable parameters.

Finally, in Fig. 4 we calculate $\sigma_{1}(\Omega)$ in LSCO (updated from Ref. [27] to incorporate the new, lower degree of unitarity scattering) and compare it to expected results for Tl-2201. The conductivity at frequency $\Omega$ is given by

$$
\begin{aligned}
\sigma_{1}= & \frac{N_{0} e^{2}}{2 \Omega} \int_{-\infty}^{\infty} d \omega(f(\omega+\Omega)-f(\omega)) \\
& \times\left\langle v_{F, x}^{2} \operatorname{Re}\left\{A_{++}-A_{+-}\right\}\right\rangle_{\mathrm{FS}},
\end{aligned}
$$

where $A_{+ \pm}=\left(\Delta_{\mathbf{k}}^{2}+\tilde{\omega}_{+} \tilde{\omega}_{ \pm}^{\prime}+Q_{+} Q_{ \pm}^{\prime}\right) /\left(Q_{+} Q_{ \pm}^{\prime}\left(Q_{+}+Q_{ \pm}^{\prime}\right)\right)$, $\omega_{ \pm}=\omega \pm i \eta, \omega_{ \pm}^{\prime}=\omega_{ \pm}(\omega+\Omega), Q_{ \pm}=\left(\Delta_{\mathbf{k}}^{2}-\tilde{\omega}_{ \pm}^{2}\right)^{1 / 2}$, and $Q_{ \pm}^{\prime}=Q_{ \pm}(\omega+\Omega)$. Vertex corrections do not appear because we have assumed zero-range scatterers. No $\mathrm{THz}$ conductivity data are yet available on the Tl-2201 system, so this may be regarded as a prediction of the theory. As can be seen, in Tl-2201 the $\Omega \rightarrow 0$ conductivity in the normal state is significantly higher and the degree of residual uncondensed spectral weight as $T \rightarrow 0$ is significantly lower, both consistent with the lower level of disorder scattering. In addition, a narrow low frequency component is clearly visible in Tl2201 at low temperatures, but is not particularly prominent in LSCO. In neither system does the gap edge correspond to any observable feature in the conductivity spectrum, although it may indirectly appear via a $4 \Delta$ feature if spin fluctuations are included in the cleaner Tl-2201 case [54]. Inclusion of forward-scattering corrections, which could be important in the Tl-2201 system, may influence these results quantitatively. 


\section{CONCLUSION}

General statements about any part of the cuprate phase diagram are a priori dangerous because cuprates consist of differing numbers of $\mathrm{CuO}_{2}$ planes per unit cell, as well as differing charge reservoir layers. The expectation, based on analysis of simple models, that correlations should weaken and that Fermi-liquid properties should become clearly observable as one overdopes, has proven difficult to verify. While normal-state quasiparticle features are now routinely observed by ARPES in some systems at both nodal and antinodal points, some classic manifestations of normal-state Fermi-liquid behavior like $T^{2}$ resistivity do not appear to be realized over significant temperature ranges [55]. This may be due to singular self-energy effects [56], but also quite likely to the presence of inelastic scattering from nearly condensed fluctuating order. In any case, in the superconducting state the quasiparticle scattering rate generally collapses, leading to well-defined Bogoliubov quasiparticles, so the possibility to observe the underlying Fermi liquid is enhanced. Our calculations show that the Landau-BCS paradigm provides an adequate description of the low energy phenomenology of the overdoped cuprates, provided the starting point is an accurate parametrization of the electronic dispersion and the occasionally nonintuitive effects of disorder are accounted for. This in turn strongly suggests that no exotic new physics should be required to understand the occurrence of superconductivity starting from a weak-coupling approach on the overdoped side.

\section{ACKNOWLEDGMENTS}

We are grateful for useful discussions with L. Benfatto, A. Damascelli, T. P. Devereaux, J. S. Dodge, D. G. Hawthorn, M. P. Kennett, S. A. Kivelson, C. Pépin, and J. E. Sonier. We gratefully acknowledge financial support from the Natural Science and Engineering Research Council of Canada, the Canadian Institute for Advanced Research, and the Canadian Foundation for Innovation. P.J.H. was partially supported by the National Science Foundation under Grant No. NSF-DMR-1849751.

\section{APPENDIX A: FERMI-SURFACE AVERAGES}

To properly capture the phenomenology of the overdoped cuprates it is essential to begin with realistic models of the energy dispersion and Fermi surface, as discussed in Ref. [26]. The Fermi-surface average is then defined as

$$
\langle\cdots\rangle_{\mathrm{FS}} \equiv \frac{1}{N_{0}} \int_{0}^{2 \pi} N_{\phi}(\cdots) d \phi,
$$

where the angle-dependent density of states is

$$
N_{\phi}=\frac{1}{2 \pi^{2} \hbar d} \frac{\left|k_{F}\right|^{2}}{\mathbf{k}_{F} \cdot \mathbf{v}_{F}} .
$$

Here $N_{0}$ is the total density of states and $d$ is the average spacing between the copper-oxide layers: $d=13.15 / 2=6.57 \AA$ for LSCO and $d=23.2 / 2=11.6 \AA$ for Tl-2201. The angledependent Fermi momentum, $\mathbf{k}_{F}$, and Fermi velocity, $\mathbf{v}_{F}$, are obtained from tight-binding parametrizations of ARPESderived energy dispersions.

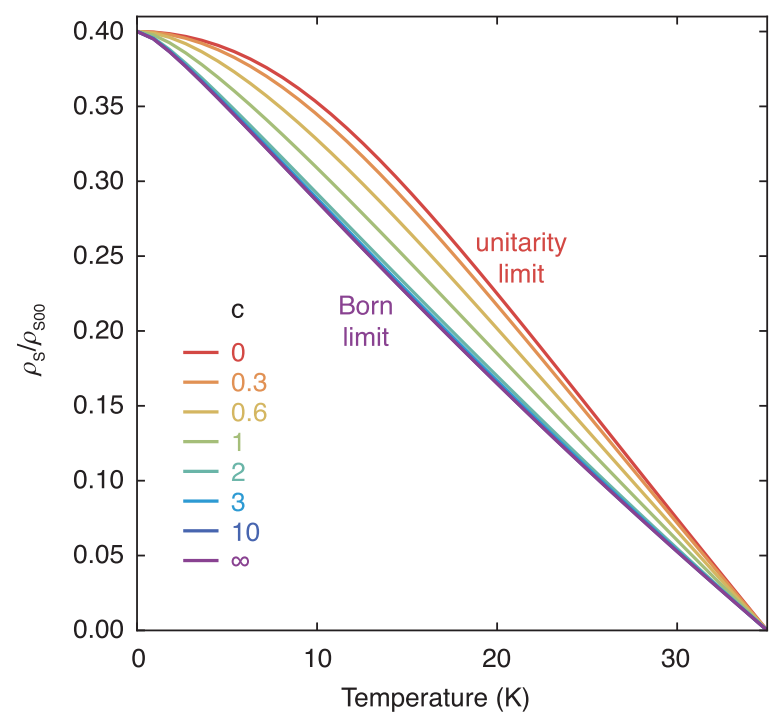

FIG. 5. Superfluid density calculated using the band structure of LSCO and a $d$-wave order parameter, for various impurity phase shifts. In each case the underlying scattering parameter $\Gamma_{N}$ has been adjusted to fix the zero-temperature superfluid fraction at $\rho_{s} / \rho_{s 00}=$ 0.4 , corresponding to the level of pair breaking in near optimally doped LSCO $[24,26]$. Note that the Born limit results are nearly indistinguishable from those of the full $t$-matrix evaluation down to a phase shift parameter of $c \simeq 2$. This illustrates the broad generality of the Born limit, which in practice extends over a wide regime of scatterer densities and scattering strengths. Details of the superfluid density calculation are given in Ref. [26].

\section{APPENDIX B: ARPES-DERIVED DISPERSIONS FOR LSCO AND Tl-2201}

For LSCO, tight-binding parametrizations of the dopingdependent Fermi surface were obtained from a series of ARPES measurements at different dopings [42]. These were the basis of our earlier calculations in Refs. [26,27] and were essential to providing an accurate account of the electrodynamic response in LSCO, in particular the nearly linear temperature dependence of superfluid density between $T=0$ and $T_{c}$ shown in Fig. 5, which is not obtained in the case of an isotropic Fermi surface. We note that the ARPES tight-binding fits in LSCO were carried out over the full bandwidth and do not capture the effects of many-body renormalization close to the Fermi level, as pointed out by the authors of Ref. [42]. As a result, the conductivity calculations in Ref. [27] required an overall renormalization of plasma frequency by a factor of 0.3 , consistent with previous work on LSCO [57].

For overdoped Tl-2201, Platé et al. [43] carried out a tight-binding fit to low energy ARPES spectra on $T_{c}=30 \mathrm{~K}$ material and obtained the dispersion

$$
\begin{aligned}
\epsilon_{\mathbf{k}}= & \mu+\frac{t_{1}}{2}\left(\cos k_{x}+\cos k_{y}\right)+t_{2} \cos k_{x} \cos k_{y} \\
& +\frac{t_{3}}{2}\left(\cos 2 k_{x}+\cos 2 k_{y}\right) \\
& +\frac{t_{4}}{2}\left(\cos 2 k_{x} \cos k_{y}+\cos k_{x} \cos 2 k_{y}\right)+t_{5} \cos 2 k_{x} \cos 2 k_{y},
\end{aligned}
$$


in which the wave vector $\mathbf{k}=\left(k_{x}, k_{y}\right)$ is measured in units of inverse lattice parameter, with hopping parameters $t_{1}=-0.725, t_{2}=0.302, t_{3}=0.0159, t_{4}=-0.0805$, and $t_{5}=0.0034 \mathrm{eV}$. In the absence of ARPES spectra at other dopings, we model the doping dependence of the dispersion by a rigid band shift, a relatively safe assumption as Tl-2201 is situated far from a van Hove crossing. In contrast to LSCO, we note that the ARPES tight-binding fits for Tl-2201 were carried out at very low energies (tens of $\mathrm{meV}$ ) and fully capture the many-body flattening of the dispersion near the Fermi level. As a result, no additional renormalization factors are required. In particular, the Tl-2201 superfluid density plotted in Fig. 1(b) and the thermal conductivity plotted in Fig. 3 are fully dimensionful quantities calculated directly from the tight-binding dispersion (B1), with no adjustable band parameters.

\section{APPENDIX C: THE BORN LIMIT AND IMPURITY PHASE SHIFT}

Most of the analysis presented here, in addition to the results in Refs. [26,27], was obtained assuming weak scatterers in the Born limit, plus a small admixture of strong scatterers. However, the argument that the dopant atoms located out of the $\mathrm{CuO}_{2}$ plane correspond to such an extremely weak scattering potential should be examined critically, particularly in the case of LSCO where the Sr dopants are located only about $2 \AA$ above the plane. In Fig. 5 we show that in terms of the dimensionless parameter $c=2 / \pi V_{\mathrm{imp}} N_{0}$, where $V_{\mathrm{imp}}$ is the impurity potential and $N_{0}$ is the total density of states at the Fermi level, there is in fact a wide range of weak to intermediate-strength scattering potentials that produce results virtually identical to the Born limit, illustrating that the Born limit is in fact a regime of broad physical applicability. Since $N_{0}$ in our ARPES-derived band structures is, respectively, 7.5 and $3.5 \mathrm{eV}^{-1}$ (per formula unit) for LSCO and Tl-2201, this means that impurities with potentials up to $\sim 0.1 \mathrm{eV}$ (formula unit) are compatible with the Born limit results presented here. An upper bound on the Sr scattering strength can be obtained by attributing all scattering to the $\mathrm{Sr}$ dopants and relating this to the normal-state elastic scattering rate $\Gamma_{N}$. Assuming that the scatterers are indeed close to the Born limit, $\Gamma_{N}=\frac{\pi}{2} n_{i} N_{0} V_{\text {imp. }}^{2}$. For overdoped LSCO, we set the Sr impurity concentration at $n_{i}=0.2$ per formula unit and, as above, a total density of states $N_{0}=7.5 \mathrm{eV}^{-1}$. With our current choice $\Gamma_{N}=18 \pi \mathrm{K}$, confirmed qualitatively by analysis of optical data in Ref. [27], we obtain $V_{\mathrm{imp}} \approx 45 \mathrm{meV}$. This establishes that the Sr dopants are indeed in the weak scattering regime. We note that this is a worst case estimate of the $\mathrm{Sr}$ scattering strength, in particular because it ignores the presence of oxygen vacancies. Indeed, preliminary $a b$ initio calculations show that $\mathrm{Sr}$ impurity potentials in LSCO are of order $35 \mathrm{meV}$, or $V_{\mathrm{imp}} N_{0} \approx 0.26$, indicating that the Born approximation is valid [58].

\section{APPENDIX D: ENTROPY-CONSERVING ANALYSIS OF RESIDUAL HEAT CAPACITY IN Tl-2201}

The best data on the doping-dependent specific heat of Tl-2201 come from the study on polycrystalline material by

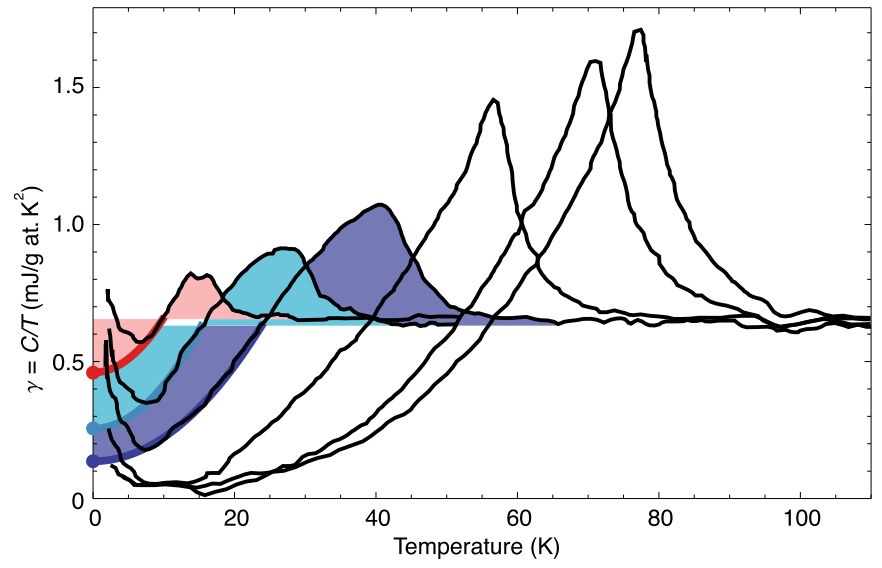

FIG. 6. Heat capacity data for overdoped Tl-2201 from Loram et al. [45], showing the entropy-conserving fits used to estimate the residual Sommerfeld coefficient at the three highest dopings (three lowest $T_{c}$ 's).

Loram et al., reported in Ref. [45]. While the differential calorimetry method works very well at high temperatures, successfully isolating the electronic specific heat from a large phonon background, clear Schottky contributions are visible at low temperature and are more difficult to subtract. To estimate the residual value of the Sommerfeld coefficient, $\gamma=C(T) / T$, we have fit to $\gamma(T)$ in such a way that the change in entropy

$$
\Delta S=\int_{0}^{T_{N}} \gamma(T) d T
$$

between $T=0$ and some $T_{N}>T_{c}$ is the same in the superconductor and normal states. These equal area constructions are plotted in Fig. 6 for the three highest dopings (three lowest $T_{c}$ 's) and allow a more reliable extrapolation beneath the Schottky contributions.

\section{APPENDIX E: QUASIPARTICLE DOPPLER SHIFT AND VORTEX STATE IN THE SEMICLASSICAL APPROXIMATION}

A Bogoliubov quasiparticle with Fermi velocity $\mathbf{v}_{\mathbf{k}}$, in a uniform superflow characterized by gauge-invariant pair momentum $\mathbf{p}_{s}=\hbar \mathbf{Q}$, experiences a Doppler shift $\frac{1}{2} \mathbf{p}_{s} \cdot \mathbf{v}_{\mathbf{k}}$. In the semiclassical approximation, it is assumed that the dominant effect of magnetic field in the vortex state is via the Doppler shift of the quasiparticle energies by the local superflow field. This is believed to be particularly important in $d$-wave superconductors, in which the nodal gap results in a significant density of delocalized excitations outside the vortex cores, and is associated with the appearance of $\sqrt{H}$ behavior (the so-called Volovik effect) in the clean limit [48,49,59]. To represent the vortex lattice, we follow Ref. [50] and use an approach that applies in the field regime $H_{c 1} \ll H \ll H_{c 2}$, where Meissner screening can be ignored. For magnetic field applied along the $\hat{\mathbf{z}}$ direction, the superflow field at position $\mathbf{r}$ takes the form

$$
\mathbf{Q}(\mathbf{r})=\left(Q_{x}, Q_{y}\right)=\sum_{i} \frac{\hat{\mathbf{z}} \times\left(\mathbf{r}-\mathbf{r}_{i}\right)}{\left(\mathbf{r}-\mathbf{r}_{i}\right)^{2}},
$$


where $\mathbf{r}_{i}$ is the center of the $i^{\text {th }}$ vortex. We are specifically interested in the cases of square and triangular (hexagonal) vortex lattices, as both are relevant to the cuprates. As in Ref. [50], we use a Fourier method to first carry out the sum over a single row of vortices. For a line of vortices centered on the origin and spaced by distance $a$ along the $x$ axis, we find the $x$ and $y$ components of the flow field to be, respectively,

$$
\begin{aligned}
Q_{1 x}(x, y) & =\frac{\pi}{a} \frac{\sinh \left(\frac{2 \pi y}{a}\right)}{\cos \left(\frac{2 \pi x}{a}\right)-\cosh \left(\frac{2 \pi y}{a}\right)}, \\
Q_{1 y}(x, y) & =-\frac{\pi}{a} \frac{\sin \left(\frac{2 \pi x}{a}\right)}{\cos \left(\frac{2 \pi x}{a}\right)-\cosh \left(\frac{2 \pi y}{a}\right)} .
\end{aligned}
$$

To obtain the flow field for the full vortex lattice, the sum over rows is carried out numerically and converges sufficiently rapidly that only a few rows in the vicinity of the point of interest need be included. In particular, the $1 / r$ divergence at the origin is captured exactly by the zeroth row. For the square vortex lattice, the final result is

$$
\begin{gathered}
Q_{x}(x, y)=\frac{2 \pi y}{a^{2}}+\sum_{n} Q_{1 x}(x, y-n a) \\
=\frac{2 \pi y}{a^{2}}+\frac{\pi}{a} \sum_{n} \frac{\sinh \left(\frac{2 \pi y}{a}-2 \pi n\right)}{\cos \left(\frac{2 \pi x}{a}\right)-\cosh \left(\frac{2 \pi y}{a}-2 \pi n\right)}, \\
Q_{y}(x, y)=\sum_{n} Q_{1 y}(x, y-n a) \\
=-\frac{\pi}{a} \sum_{n} \frac{\sin \left(\frac{2 \pi x}{a}\right)}{\cos \left(\frac{2 \pi x}{a}\right)-\cosh \left(\frac{2 \pi y}{a}-2 \pi n\right)},
\end{gathered}
$$

where $n$ is a set of integers and the linear term in $Q_{x}$ is a correction arising from the long-range nature of $Q_{1 x}$. For the triangular (hexagonal) vortex lattice

$$
\begin{aligned}
& Q_{x}(x, y)=\frac{4 \pi y}{\sqrt{3} a^{2}}+\sum_{n} Q_{1 x}(x-n a / 2, y-\sqrt{3} n a / 2) \\
& =\frac{4 \pi y}{\sqrt{3} a^{2}}+\frac{\pi}{a} \sum_{n} \frac{\sinh \left(\frac{2 \pi y}{a}-\sqrt{3} n \pi\right)}{\cos \left(\frac{2 \pi x}{a}-n \pi\right)-\cosh \left(\frac{2 \pi y}{a}-\sqrt{3} n \pi\right)} \\
& Q_{y}(x, y)=\sum_{n} Q_{1 y}(x-n a / 2, y-\sqrt{3} n a / 2) \\
& =-\frac{\pi}{a} \sum_{n} \frac{\sin \left(\frac{2 \pi x}{a}-n \pi\right)}{\cos \left(\frac{2 \pi x}{a}-n \pi\right)-\cosh \left(\frac{2 \pi y}{a}-\sqrt{3} n \pi\right)}
\end{aligned}
$$

The small-angle neutron scattering experiments in Ref. [46] show that the vortex lattice in overdoped LSCO adopts a square structure above a field of $0.4 \mathrm{~T}$, with primitive vectors aligned along the $\mathrm{Cu}-\mathrm{O}$ bond directions. Such a square lattice has therefore been used in the calculations of field-dependent specific heat shown in Fig. 2(c). The results for a triangular (hexagonal) vortex lattice are qualitatively similar but approximately 3-5\% larger.

To take into account order parameter suppression near the vortex core, we employ the Clem model [60], in which the normalized order parameter takes the form

$$
\frac{\Delta(\rho)}{\Delta_{\infty}}=\frac{\rho}{\left(\rho^{2}+\xi_{v}^{2}\right)^{1 / 2}} .
$$

Here $\Delta_{\infty}$ is the gap magnitude in the uniform limit, obtained from solution of the gap equation (3), in zero field, and $\rho$ is the radial displacement from the vortex center. In stronglytype-II materials, the variational core radius parameter $\xi_{v} \rightarrow$ $\sqrt{2} \xi$ [60], allowing its value to be set from magnetoresistive measurements of upper critical field in LSCO [61].

Spatial averages over the vortex-lattice unit cell are carried out by Monte Carlo integration, using a total of 50000 samples for each field point, based on pseudorandom Halton sequences generated from randomly chosen coprime pairs. The Halton sequences provide more uniform sampling and faster convergence than purely random Monte Carlo. At each sampling point in the vortex-lattice unit cell, a full Fermisurface integral is then carried out, rather than the usual nodal approximation, in order to accurately evaluate the density of states in the local superflow.

In Fig. 2(c) it is interesting that the coefficient $A$ of a $C(T \rightarrow 0, H) / T=A \sqrt{H}$ term fit to the Wang et al. [44] data on overdoped LSCO gives a nonmonotonic variation of $A$ with $x$, as does the theory. This can be possibly understood as an initial increase of the vortex core size as $\Delta$ decreases, together with a subsequent suppression of $A$ due to disorder. However, the agreement in the figure is only qualitative, not quantitative. While we have gone to some lengths to perform the most accurate semiclassical calculations appropriate to the systems studied, there is some reason to doubt that quantitative results including weak disorder can be obtained in this limit. The Volovik effect is a zero-temperature property valid for $H_{c 1} \ll H \ll H_{c 2}$. At low temperatures and energies, the mean free path of a quasiparticle experiencing Born scattering may become very long, larger than the intervortex spacing, such that the implicit assumption of a local self-energy in Eq. (6) is no longer appropriate. Furthermore, we are extending the theory to significant fractions of $H_{c 2}$ for the more overdoped cases. A more complete treatment at high fields along the lines of the Brandt-Pesch-Tewordt theory [62] may be required to describe these data accurately.
[1] B. Keimer, S. A. Kivelson, M. R. Norman, S. Uchida, and J. Zaanen, From quantum matter to high-temperature superconductivity in copper oxides, Nature (London) 518, 179 (2015).

[2] T. Timusk and B. Statt, The pseudogap in high-temperature superconductors: An experimental survey, Rep. Prog. Phys. 62, 61 (1999).
[3] M. Gurvitch and A. T. Fiory, Resistivity of $\mathrm{La}_{2-x} \mathrm{Sr}_{x} \mathrm{CuO}_{4}$ and $\mathrm{YBa}_{2} \mathrm{Cu}_{3} \mathrm{O}_{7}$ to $1100 \mathrm{~K}$ : Absence of Saturation and its Implications, Phys. Rev. Lett. 59, 1337 (1987).

[4] G. A. Thomas, J. Orenstein, D. H. Rapkine, M. Capizzi, A. J. Millis, R. N. Bhatt, L. F. Schneemeyer, and J. V. Waszczak, $\mathrm{Ba}_{2} \mathrm{YCu}_{3} \mathrm{O}_{7-\delta}$ : Electrodynamics of Crystals with High Reflectivity, Phys. Rev. Lett. 61, 1313 (1988). 
[5] C. M. Varma, P. B. Littlewood, S. Schmitt-Rink, E. Abrahams, and A. E. Ruckenstein, Phenomenology of the Normal State of Cu-O High-Temperature Superconductors, Phys. Rev. Lett. 63, 1996 (1989).

[6] J. M. Tranquada, B. J. Sternlieb, J. D. Axe, Y. Nakamura, and S. Uchida, Evidence for stripe correlations of spins and holes in copper-oxide superconductors, Nature (London) 375, 561 (1995).

[7] B. Lake, G. Aeppli, K. N. Clausen, D. F. McMorrow, K. Lefmann, N. E. Hussey, N. Mangkorntong, M. Nohara, H. Takagi, T. E. Mason, and A. Schröder, Spins in the vortices of a high-temperature superconductor, Science 291, 1759 (2001).

[8] S. A. Kivelson, I. P. Bindloss, E. Fradkin, V. Oganesyan, J. M. Tranquada, A. Kapitulnik, and C. Howald, How to detect fluctuating stripes in the high-temperature superconductors, Rev. Mod. Phys. 75, 1201 (2003).

[9] S. Sachdev, Colloquium: Order and quantum phase transitions in the cuprate superconductors, Rev. Mod. Phys. 75, 913 (2003).

[10] R. Balian and N. R. Werthamer, Superconductivity with pairs in a relative $p$ wave, Phys. Rev. 131, 1553 (1963).

[11] P. Arberg, M. Mansor, and J. P. Carbotte, Penetration depth for a 2D $d$-wave superconductor, Solid State Commun. 86, 671 (1993).

[12] P. J. Hirschfeld and N. Goldenfeld, Effect of strong scattering on the low temperature penetration depth of a $d$-wave superconductor, Phys. Rev. B 48, 4219 (1993).

[13] P. J. Hirschfeld, W. O. Putikka, and D. J. Scalapino, Microwave Conductivity of $d$-Wave Superconductors, Phys. Rev. Lett. 71, 3705 (1993).

[14] P. J. Hirschfeld, W. O. Putikka, and D. J. Scalapino, $d$-wave model for microwave response of high- $T_{c}$ superconductors, Phys. Rev. B 50, 10250 (1994).

[15] A. A. Abrikosov and L. P. Gor'kov, Contribution to the theory of superconducting alloys with paramagnetic impurities, Zh. Eksp. Teor. Fiz. 39, 1781 (1960) [Sov. Phys. JETP 12, 1243 (1961)].

[16] E. W. Huang, D. J. Scalapino, T. A. Maier, B. Moritz, and T. P. Devereaux, Decrease of $d$-wave pairing strength in spite of the persistence of magnetic excitations in the overdoped Hubbard model, Phys. Rev. B 96, 020503(R) (2017).

[17] A. V. Balatsky, I. Vekhter, and J.-X. Zhu, Impurity-induced states in conventional and unconventional superconductors, Rev. Mod. Phys. 78, 373 (2006).

[18] F. Rullier-Albenque, H. Alloul, F. Balakirev, and C. Proust, Disorder, metal-insulator crossover and phase diagram in high$T_{c}$ cuprates, Europhys. Lett. 81, 37008 (2008).

[19] P. W. Anderson, The resonating valence bond state in $\mathrm{La}_{2-x} \mathrm{Sr}_{x} \mathrm{CuO}_{4}$ and superconductivity, Science 235, 1196 (1987).

[20] Y. Ohta, T. Tohyama, and S. Maekawa, Apex oxygen and critical temperature in copper oxide superconductors: Universal correlation with the stability of local singlets, Phys. Rev. B 43, 2968 (1991).

[21] E. Pavarini, I. Dasgupta, T. Saha-Dasgupta, O. Jepsen, and O. K. Andersen, Band-Structure Trend in Hole-Doped Cuprates and Correlation with $T_{c \max }$, Phys. Rev. Lett. 87, 047003 (2001).

[22] H. Sakakibara, H. Usui, K. Kuroki, R. Arita, and H. Aoki, Two-Orbital Model Explains the Higher Transition Temperature of the Single-Layer Hg-Cuprate Superconductor Compared to that of the La-Cuprate Superconductor, Phys. Rev. Lett. 105, 057003 (2010)

[23] K. Fujita, T. Noda, K. M. Kojima, H. Eisaki, and S. Uchida, Effect of Disorder Outside the $\mathrm{CuO}_{2}$ Planes on $T_{c}$ of Copper Oxide Superconductors, Phys. Rev. Lett. 95, 097006 (2005).

[24] I. Božović, X. He, J. Wu, and A. T. Bollinger, Dependence of the critical temperature in overdoped copper oxides on superfluid density, Nature (London) 536, 309 (2016).

[25] F. Mahmood, X. He, I. Bozovic, and N. P. Armitage, Locating the Missing Superconducting Electrons in Overdoped Cuprates, Phys. Rev. Lett. 122, 027003 (2019).

[26] N. R. Lee-Hone, J. S. Dodge, and D. M. Broun, Disorder and superfluid density in overdoped cuprate superconductors, Phys. Rev. B 96, 024501 (2017).

[27] N. R. Lee-Hone, V. Mishra, D. M. Broun, and P. J. Hirschfeld, Optical conductivity of overdoped cuprate superconductors: Application to $\mathrm{La}_{2-x} \mathrm{Sr}_{x} \mathrm{CuO}_{4}$, Phys. Rev. B 98, 054506 (2018).

[28] B. Vignolle, A. Carrington, R. A. Cooper, M. M. J. French, A. P. Mackenzie, C. Jaudet, D. Vignolles, C. Proust, and N. E. Hussey, Quantum oscillations in an overdoped high- $T_{c}$ superconductor, Nature (London) 455, 952 (2008).

[29] A. F. Bangura, P. M. C. Rourke, T. M. Benseman, M. Matusiak, J. R. Cooper, N. E. Hussey, and A. Carrington, Fermi surface and electronic homogeneity of the overdoped cuprate superconductor $\mathrm{Tl}_{2} \mathrm{Ba}_{2} \mathrm{CuO}_{6+\delta}$ as revealed by quantum oscillations, Phys. Rev. B 82, 140501(R) (2010).

[30] P. M. C. Rourke, A. F. Bangura, T. M. Benseman, M. Matusiak, J. R. Cooper, A. Carrington, and N. E. Hussey, A detailed de Haas-van Alphen effect study of the overdoped cuprate $\mathrm{Tl}_{2} \mathrm{Ba}_{2} \mathrm{CuO}_{6+\delta}$, New J. Phys. 12, 105009 (2010).

[31] A. P. Mackenzie, S. R. Julian, G. G. Lonzarich, A. Carrington, S. D. Hughes, R. S. Liu, and D. C. Sinclair, Resistive Upper Critical-Field of $\mathrm{Tl}_{2} \mathrm{Ba}_{2} \mathrm{CuO}_{6}$ at Low-Temperature and High Magnetic-Fields, Phys. Rev. Lett. 71, 1238 (1993).

[32] C. Proust, E. Boaknin, R. W. Hill, L. Taillefer, and A. P. Mackenzie, Heat Transport in a Strongly Overdoped Cuprate: Fermi Liquid and a Pure $d$-Wave BCS Superconductor, Phys. Rev. Lett. 89, 147003 (2002).

[33] N. E. Hussey, M. Abdel-Jawad, A. Carrington, A. P. Mackenzie, and L. Balicas, A coherent three-dimensional Fermi surface in a high-transition-temperature superconductor, Nature (London) 425, 814 (2003).

[34] P. Hirschfeld, D. Vollhardt, and P. Wölfle, Resonant impurity scattering in heavy fermion superconductors, Solid State Commun. 59, 111 (1986).

[35] S. Schmitt-Rink, K. Miyake, and C. M. Varma, Transport and Thermal Properties of Heavy-Fermion Superconductors: A Unified Picture, Phys. Rev. Lett. 57, 2575 (1986).

[36] V. G. Kogan, R. Prozorov, and V. Mishra, London penetration depth and pair breaking, Phys. Rev. B 88, 224508 (2013).

[37] D. Deepwell, D. C. Peets, C. J. S. Truncik, N. C. Murphy, M. P. Kennett, W. A. Huttema, R. Liang, D. A. Bonn, W. N. Hardy, and D. M. Broun, Microwave conductivity and superfluid density in strongly overdoped $\mathrm{Tl}_{2} \mathrm{Ba}_{2} \mathrm{CuO}_{6+\delta}$, Phys. Rev. B 88, 214509 (2013).

[38] J. H. Brewer, S. L. Stubbs, R. Liang, D. A. Bonn, W. N. Hardy, J. E. Sonier, W. A. MacFarlane, and D. C. Peets, Signatures of new $d$-wave vortex physics in overdoped $\mathrm{Tl}_{2} \mathrm{Ba}_{2} \mathrm{CuO}_{6+x}$ revealed by TF- $\mu^{+}$SR, Sci. Rep. 5, 14156 (2015). 
[39] Ch. Niedermayer, C. Bernhard, U. Binninger, H. Glückler, J. L. Tallon, E. J. Ansaldo, and J. I. Budnick, Muon Spin Rotation Study of the Correlation between $T_{c}$ and $n_{s} / m^{*}$ in Overdoped $\mathrm{Tl}_{2} \mathrm{Ba}_{2} \mathrm{CuO}_{6+\delta}$, Phys. Rev. Lett. 71, 1764 (1993).

[40] Y. J. Uemura, A. Keren, L. P. Le, G. M. Luke, W. D. Wu, Y. Kubo, T. Manako, Y. Shimakawa, M. Subramanian, J. L. Cobb, and J. T. Markert, Magnetic-field penetration depth in $\mathrm{Tl}_{2} \mathrm{Ba}_{2} \mathrm{CuO}_{6+\delta}$ in the overdoped regime, Nature (London) 364, 605 (1993).

[41] Y. Shimakawa, Chemical and structural study of tetragonal and orthorhombic $\mathrm{Tl}_{2} \mathrm{Ba}_{2} \mathrm{CuO}_{6}$, Physica C 204, 247 (1993).

[42] T. Yoshida, X. J. Zhou, K. Tanaka, W. L. Yang, Z. Hussain, Z. X. Shen, A. Fujimori, S. Sahrakorpi, M. Lindroos, R. S. Markiewicz, A. Bansil, S. Komiya, Y. Ando, H. Eisaki, T. Kakeshita, and S. Uchida, Systematic doping evolution of the underlying Fermi surface of $\mathrm{La}_{2-x} \mathrm{Sr}_{x} \mathrm{CuO}_{4}$, Phys. Rev. B 74, 224510 (2006).

[43] M. Platé, J. D. F. Mottershead, I. S. Elfimov, D. C. Peets, R. Liang, D. A. Bonn, W. N. Hardy, S. Chiuzbaian, M. Falub, M. Shi, L. Patthey, and A. Damascelli, Fermi Surface and Quasiparticle Excitations of Overdoped $\mathrm{Tl}_{2} \mathrm{Ba}_{2} \mathrm{CuO}_{6+\delta}$, Phys. Rev. Lett. 95, 077001 (2005).

[44] Y. Wang, J. Yan, L. Shan, H.-H. Wen, Y. Tanabe, T. Adachi, and Y. Koike, Weak-coupling $d$-wave BCS superconductivity and unpaired electrons in overdoped $\mathrm{La}_{2-x} \mathrm{Sr}_{x} \mathrm{CuO}_{4}$ single crystals, Phys. Rev. B 76, 064512 (2007).

[45] J. W. Loram, K. A. Mirza, J. M. Wade, J. R. Cooper, and W. Y. Liang, The electronic specific heat of cuprate superconductors, Physica C 235-240, 134 (1994).

[46] J. Chang, J. S. White, M. Laver, C. J. Bowell, S. P. Brown, A. T. Holmes, L. Maechler, S. Strässle, R. Gilardi, S. Gerber et al., Spin density wave induced disordering of the vortex lattice in superconducting $\mathrm{La}_{2-x} \mathrm{SR}_{x} \mathrm{CuO}_{4}$, Phys. Rev. B 85, 134520 (2012).

[47] P. J. Hirschfeld, P. Wölfle, and D. Einzel, Consequences of resonant impurity scattering in anisotropic superconductors: Thermal and spin relaxation properties, Phys. Rev. B 37, 83 (1988).

[48] G. E. Volovik, Superconductivity with lines of gap nodes: Density of states in the vortex, Pis'ma Zh. Eksp. Teor. Fiz. 58, 457 (1993) [JETP Lett. 58, 469 (1993)].

[49] C. Kübert and P. J. Hirschfeld, Vortex contribution to specific heat of dirty $d$-wave superconductors: Breakdown of scaling, Solid State Commun. 105, 459 (1998).

[50] L. B. Ioffe and A. J. Millis, $d$-wave superconductivity in doped Mott insulators, J. Phys. Chem. Solids 63, 2259 (2002).

[51] J. Takeya, Y. Ando, S. Komiya, and X. F. Sun, LowTemperature Electronic Heat Transport in $\mathrm{La}_{2-x} \mathrm{Sr}_{x} \mathrm{CuO}_{4}$ Sin- gle Crystals: Unusual Low-Energy Physics in the Normal and Superconducting States, Phys. Rev. Lett. 88, 077001 (2002).

[52] M. Sutherland, D. G. Hawthorn, R. W. Hill, F. Ronning, S. Wakimoto, H. Zhang, C. Proust, E. Boaknin, C. Lupien, L. Taillefer, R.-X. Liang, D. A. Bonn, W. N. Hardy, R. Gagnon, N. E. Hussey, T. Kimura, M. Nohara, and H. Takagi, Thermal conductivity across the phase diagram of cuprates: Low-energy quasiparticles and doping dependence of the superconducting gap, Phys. Rev. B 67, 174520 (2003).

[53] D. G. Hawthorn, S. Y. Li, M. Sutherland, E. Boaknin, R. W. Hill, C. Proust, F. Ronning, M. A. Tanatar, J. Paglione, L. Taillefer, D. Peets, R. Liang, D. A. Bonn, W. N. Hardy, and N. N. Kolesnikov, Doping dependence of the superconducting gap in $\mathrm{Tl}_{2} \mathrm{Ba}_{2} \mathrm{CuO}_{6+\delta}$ from heat transport, Phys. Rev. B 75, 104518 (2007).

[54] S. M. Quinlan, P. J. Hirschfeld, and D. J. Scalapino, Infrared conductivity of a $d_{x^{2}-y^{2}}$-wave superconductor with impurity and spin-fluctuation scattering, Phys. Rev. B 53, 8575 (1996).

[55] L. Taillefer, Scattering and pairing in cuprate superconductors, Annu. Rev. Condens. Matter Phys. 1, 51 (2010).

[56] K. Miyake and C. M. Varma, Renormalizations in unconventional superconducting states born of normal and singular Fermi liquids, Phys. Rev. B 98, 174501 (2018).

[57] W. J. Padilla, Y. S. Lee, M. Dumm, G. Blumberg, S. Ono, K. Segawa, S. Komiya, Y. Ando, and D. N. Basov, Constant effective mass across the phase diagram of high- $T_{c}$ cuprates, Phys. Rev. B 72, 060511(R) (2005).

[58] T. Berlijn (private communication).

[59] K. A. Moler, D. J. Baar, J. S. Urbach, R. Liang, W. N. Hardy, and A. Kapitulnik, Magnetic Field Dependence of the Density of States of $\mathrm{YBa}_{2} \mathrm{Cu}_{3} \mathrm{O}_{6.95}$ As Determined from the Specific Seat, Phys. Rev. Lett. 73, 2744 (1994).

[60] J. R. Clem, Simple model for the vortex core in a type II superconductor, J. Low Temp. Phys. 18, 427 (1975).

[61] P. M. C. Rourke, I. Mouzopoulou, X. Xu, C. Panagopoulos, Y. Wang, B. Vignolle, C. Proust, E. V. Kurganova, U. Zeitler, Y. Tanabe, T. Adachi, Y. Koike, and N. E. Hussey, Phasefluctuating superconductivity in overdoped $\mathrm{La}_{2-x} \mathrm{Sr}_{x} \mathrm{CuO}_{4}$, Nat. Phys. 7, 455 (2011).

[62] U. Brandt, W. Pesch, and L. Tewordt, Theory of the density of states of pure type-II superconductors in high magnetic fields, Z. Phys. 201, 209 (1967).

Correction: A support statement in the Acknowledgments contained an error and has been fixed. 